\title{
Drowsy Driver Detection Based on Eye by Using Digital Image Processing
}

\author{
Sheena Chopra, S. R. Dogiwal \\ Department of Computer Science \& Engineering,Swami Keshvanand Institute of Technology, Management \\ \& Gramothan Jaipur-302017 (INDIA) \\ Email-dogiwal@gmail.com \\ Received 11.10.2019 received in revised form 09.10.2020, accepted 10.10.2020
}

\begin{abstract}
This paper focuses on driver drowsiness detection based on eye by using Digital Image processing technique. The proposed system is a non intrusive driver drowsiness monitoring system that is able to detect drowsiness of driver in wearing spectacles and dim light level. The proposed system is implemented or tested by using webcam to capture image then face and eye detection techniques used. The intensity of eye blinking area is checked to determine whether eye is close or open. An alarm is made on eye close condition. The samples are collected from left side, right side and with spectacles. The accuracy of the system is $98 \%$ and time taken to perform a sample is 30-50 seconds. Which is quite satisfactory.
\end{abstract}

Keywords :Digital Image Processing, MATLAB, Face recognition.

\section{INTRODUCTION}

Drowsiness is one of the difficult issue in road mishaps. The casualty rate due sluggishness is exceptionally higher. The worldwide status give an account of road security distributed by the WHO recognized primary driver of road accident are because of driver slip-ups and lack of regard. A mishap including driver sluggishness has a high casualty rate on the grounds that the perception, affirmation and vehicle control capacities lessen forcefully while driver nodding off. It is discovered that laziness is one the central point that causes latency of the driver. It prompts expanded in number of road mishaps every year. On the off chance that sluggishness is identified sufficiently early, at that point it could spare numerous road accident. The developing number of mishap in world as of late has turned into an issue of genuine worry for the general public, so mishaps must be forestalled before they occur and this thing relies on the driver. Anticipating mishaps brought about by sluggishness requires a procedure for distinguishing languor in a driver and a method for exciting the driver from that lethargic condition. The venture portrays a framework that utilizes a picture handling method to perceive the open or shut condition of the driver's eyes as a method for identifying laziness in the driver's seat. The anodes that were connected to the body of the driver were tiring and uncomfortable for the driver. The driverfeel uneasy and ready to focus on driving because of this. The cathodes required time to time substitution. There were some different frameworks additionally that were actualized to distinguish the sluggishness of the driver utilized GSR, ECG, EMG. These framework additionally required the connection of the sensors to the body of the driver.

\section{REVIEW LITERATURE}

The literature review also tells existing work and describe strength and weakness of drowsy driver detection techniques.

Kumari Kusuma B.M., Kumar Ramakanth P., 2017 presented a survey on Drowsy Driver Detection System. They discussed about subjective, vehicle, behaviour, physiological, hybrid based measures for drowsiness detection system [1].

Oraan Khunpisuth, et-al, 2016 proposed work used Raspberry Pi 3 Model B Raspberry Pi camera to acquire the image. This system work on face,eye blinking and head level. The Haar Cascade Classifier was used in different light conditions. The problem was solved by using geometric rotation. [2].

Chellappa Yogesh, et-al, 2016 explained about driver fatigue detection system. They considered both physiological and physical signs. Physiological factors included like body temperature and pulse rate and physical parameters were included yawning, drooping eyelids, and blinks duration. The accuracy was about $80.55 \%$. [3].

Ahmed Javed, et-al, 2015 proposed a non-intrusive computer based idea to detect drowsiness of driver. The small camera was used to get the driver's image. The histogram equalization method used to adjust contrast and Haar Cascade is used to detect the features from the image. The reflection of retina was used to check whether eye was closed or not [4]

Kumar Madhan A, et-al, 2019 proposed drowsy driving warning and traffic collision information system using IoT system. In this system Haar 
Cascaded algorithm was used for eye detection. An IoT based module was used to get location information and to send message. WiFi module was used to update location information in the server [5].

\section{PROPOSED SYSTEM}

At first the images are recorded using the Webcam. Further picture changed over in Gray Scale format. After converting in gray scale format first get faces from images. Different face detection methods are available. In proposed work Paul Viola and Michael Jones algorithm for face detection are used [6]. The valuable part which can be said ROI, is cropped. It is observed that if eye is open then white part of this cropped block having some high intensity value comparatively black part and it show some high mean value, if eye is close then there will be only skin part ( no white part will be there) and it show some low mean value. This method will decide eye is open or close. A alarm is issued to driver.

\subsection{Flow Chart of the proposed work}

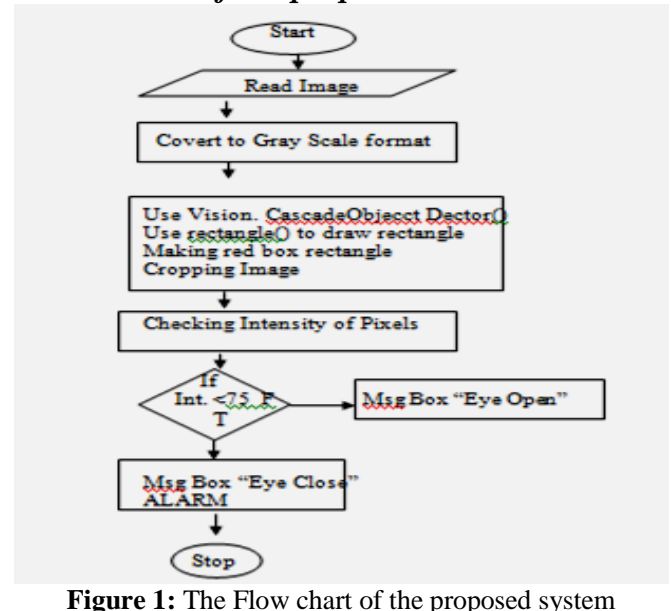

Whole functioning of the system is depicted in above flow chart. The first step to read / input image by using camera then it is converted to gray scale format. Again input choice for checking left or right eye. Now apply Haar Cascade Classifier and separate facial feature from the image like eye. After that crop eye block and computer mean intensity value of that block, the mean intensity value decide whether eye is open or close.

\subsection{Step by Step Procedure}

Step 1. INPUT Image

Step2. CHANGE IMAGE to Gray Scale

Step3. INPUT CHOICE (1 for left and 2 for Right)

Step4. IF CHOICE ==1

Step5. COMPUTEvision.CascadeObjectDetector
Step6. CROP EYE BLOCK

Step7. COMPUTE MEAN INTENSITY

Step8. IF MEAN INTENSITY < 75

MSGBOX ("EYE Closed") and ALARM

ELSE MSGBOX ("EYE Is Open")

a. COMPUTE vision.CascadeObjectDetector

b. CROP EYE BLOCK

c. COMPUTE MEAN INTENSITY

IF MEAN INTENSITY $<75$

MSGBOX ("EYE Closed") andALARM

ELSE MSGBOX ("EYE Is Open”)

END IF

END IF

Step 9.STOP

\subsection{Proposed Work}

This segment decides the basic steps or functioning of proposed work. We perform the task by executing one by one step.

3.3.1 Image Acquisition: In this the images are captured by using Webcam and directly transferred to $\mathrm{PC}$ in to a specific folder.

3.3.2 Face and Eyes Detection:After getting the image, human face is detected. The proposed work is based on "Rapid Object Detection using a Boosted Cascade of Simple Feature" [6], it is used by Paul Viola and Michael Jones. The method efficiently detects faces from an image with good accuracy rate. This algorithm work on 4 stages like Haar Feature Selection, Creating an Integral Image, Adaboost Training, Cascading Classifiers [6].

3.3.3 Region of Interest: Region of Interest (ROI) is detected from complete image. The ROI area first obtain by using Haar Cascade Classifier which include height, width. This region shown by fig.2.

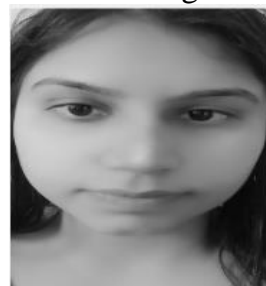

Figure2 : The region of Interest of an image

3.3.4 Eye Detection: After obtaining face of the driver, eyes part of image is separated by Paul Viola and Michael Jones algorithm for face detection [6]. This method marks both eyes with rectangle colored boxes. To equalize various face sizes, images are resized in same dimensions. The area of the eye is further cropped.

3.3.5 Drowsiness detection: It is observed that when eyes are opened then having some white portion on image which area have some high intensity value, if eyes are closed then having same equal dark area which have some low intensity value comparatively white portion. The mean value of open eye block will be certainly higher than close eye block. After 
obtaining eye block the mean value of that block is checked, if it is greater than a particular value than eye is open otherwise detected eye is closed. The proposed system collected more than 100 samples to test the system, it is observed high accuracy in minimum process time.
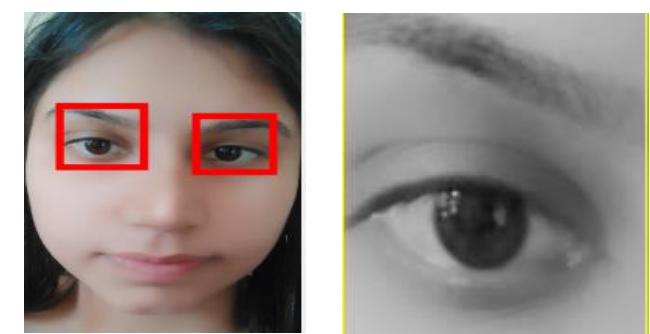

Figure 3: Eyes Detection with rectangle mark and cropped eye

\section{EXPERIMENTAL RESULTS AND OUTPUTS}

The propose system collected more 100 samples and checked its efficiency on all levels. The results are calculated at own collected data and ready available data on web.

4.1 Acquisition of Image: Image of face is captured by web camera. This camera is able to capture all facial activities of the driver.

4.2 Grayscale Conversion: The image which is captured by camera is converted in grayscale format. Now each pixel of image is available as array in form of intensity values.

4.3 Region of Interest:After getting complete image of driver, the face is detected as region of interest. The Haar Cascade Classifier is used to detect face and eye of whole image.

4.4 Cropping of Eye Area of facial Image: After finding eye area of an image, there is task to crop both eye part from the face. This task is done by using imcrop() function in matlab and when we see intensity value of this area, it looks like fig. 4. It is observed that intensity value of each pixel in eye block image in center part of open eye condition (which has white part) having some high intensity value and other part of eye block having some low intensity value as shown in Fig. 4

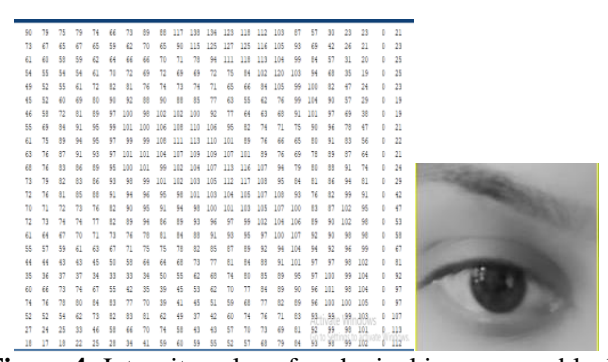

Figure 4: Intensity value of each pixel in open eye block
4.5 Experimental results of Accuracy, Process Time and Mean Intensity Value: The proposed system collected 100 samples and 30 sample were used to find the experimental results.

4.6 Mean Intensity Value of Samples: The proposed system was examined on 30 samples the mean Intensity value of cropped eye block calculated in both states.

Mean Intensity Value $=\frac{\sum \text { Intensity Value of Each Pixel }}{\text { Total No.of Pixel }}$

Mean Intensity Value $=\frac{\sum_{i=0, j=0}^{m, n} \mathrm{Pij}}{N}$

4.7 GraphicaRepresentation: The graphs can be drawn between mean intensity value of close eye condition and open eye condition from different views.

\subsection{Front View and close Eye View}

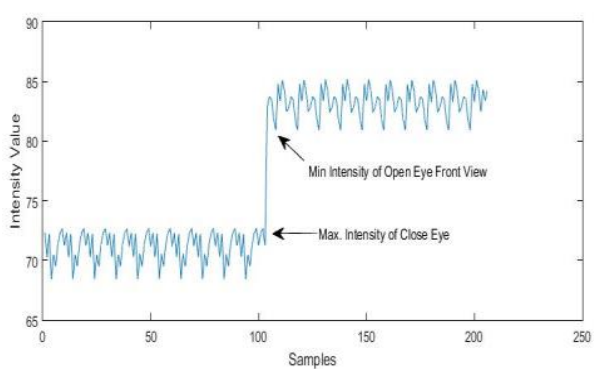

Figure 5: Graphical representation of Close Eye and Front View Open Eye

The above graph show that maximum mean intensity value of close eye condition is below 72 and minimum mean intensity value of open eye front view is 81 . It is observed that there is big gap between close eye and open eye front view. This gap decide open and close eye condition.

4.9Left Eye view and Close Eye

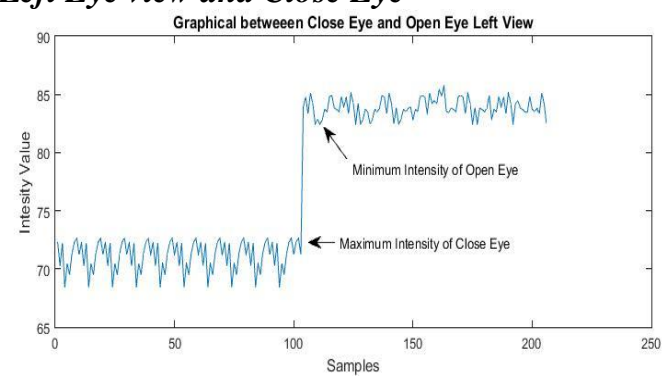

Figure 6: Graphical representation of Close Eye and Left View Open Eye

The above graph show that maximum mean intensity value of close eye condition is below 72 and minimum mean intensity value of open eye left view is 81 . It is observed that there is big gap between close eye and open eye front view. This gap decide open and close eye condition. 
4.10 Right View and Close Eye View:

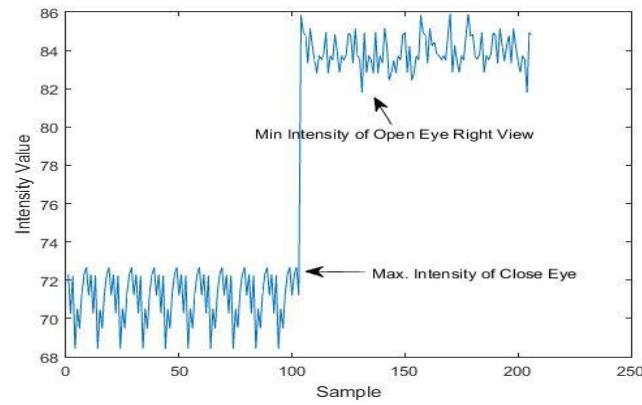

Figure7: Graphical representation of Close Eye and Right View Open Eye

The above graph show that maximum mean intensity value of close eye condition is below 72 and minimum mean intensity value of open eye right view is 81 . It is observed that there is big gap between close eye and open eye front view. This gap decide open and close eye condition.

4.11 Accuracy of Each Sample: It is observed that accuracy of each sample on each attempt is about true.

Accuracy in percentage $=\frac{\text { Total Number True chance }}{\text { Total Number of Chance }} \mathrm{X}$ 100

Accuracy in percentage $=(98 / 100) \times 100=98.00$

Hence performance of system is quite satisfactory. The experiments were performed for both spectacles or without spectacles.

Table 1: shows the comparative analysis of proposed work with other methods. It also shows that proposed work is having good and satisfactory result on synthetics data.

\begin{tabular}{|c|c|c|c|}
\hline $\begin{array}{c}\text { Ashish } \\
\text { Kumar,Rush } \\
\text { a [patra[1] }\end{array}$ & $\begin{array}{c}\text { Image } \\
\text { acquisition } \\
\text { device }\end{array}$ & Webcam & Non intrusive \\
\hline $\begin{array}{c}\text { Oraan } \\
\text { Khunpisuth, } \\
\text { Narit } \\
\text { Hnoohom[3] }\end{array}$ & $\begin{array}{c}\text { Raspberry } \\
\text { Pi Camera }\end{array}$ & Non intrusive & $99 \%$ \\
\hline $\begin{array}{c}\text { Yogesh } \\
\text { Chellappa, } \\
\text { Narendra } \\
\text { Nath }\end{array}$ & Camera & Combination \\
of both & $85 \%$ \\
\hline $\begin{array}{c}\text { Joshi[4] } \\
\text { Arun D } \\
\text { Panicker, } \\
\text { Madhu S } \\
\text { Nair[10] }\end{array}$ & Camera & Non-intrusive & $93 \%$ \\
\hline $\begin{array}{c}\text { Proposed } \\
\text { research } \\
\text { work }\end{array}$ & Webcam & Non-intrusive & $98 \%$ \\
\hline
\end{tabular}

4.11 Summary of Results: It is observed that proposed system is designed on working of only one parameter and provide more than 98\% percentage accuracy. The time taken to process each sample was less than single minute. The mean intensity value in close eye condition is less than 75 and in minimum intensity value in open eye condition is 81 . This cut off can be used to detect the drowsiness of eye. The findings of proposed work is good and satisfactory.

4.12 Comparative Analysis :The proposed algorithm is tested on INVEDRIFAC data set.[12] The videos and images of this concern are downloaded from site. The proposed algorithm has been tested on 100 image from different views for each sample. It is found that system give negligible error/ false value.

4.13 Demonstration of Proposed Work:Five sample views from different angles shown in below

\begin{tabular}{|l|l|}
\hline \multicolumn{3}{|c|}{ Front View } \\
\hline
\end{tabular}




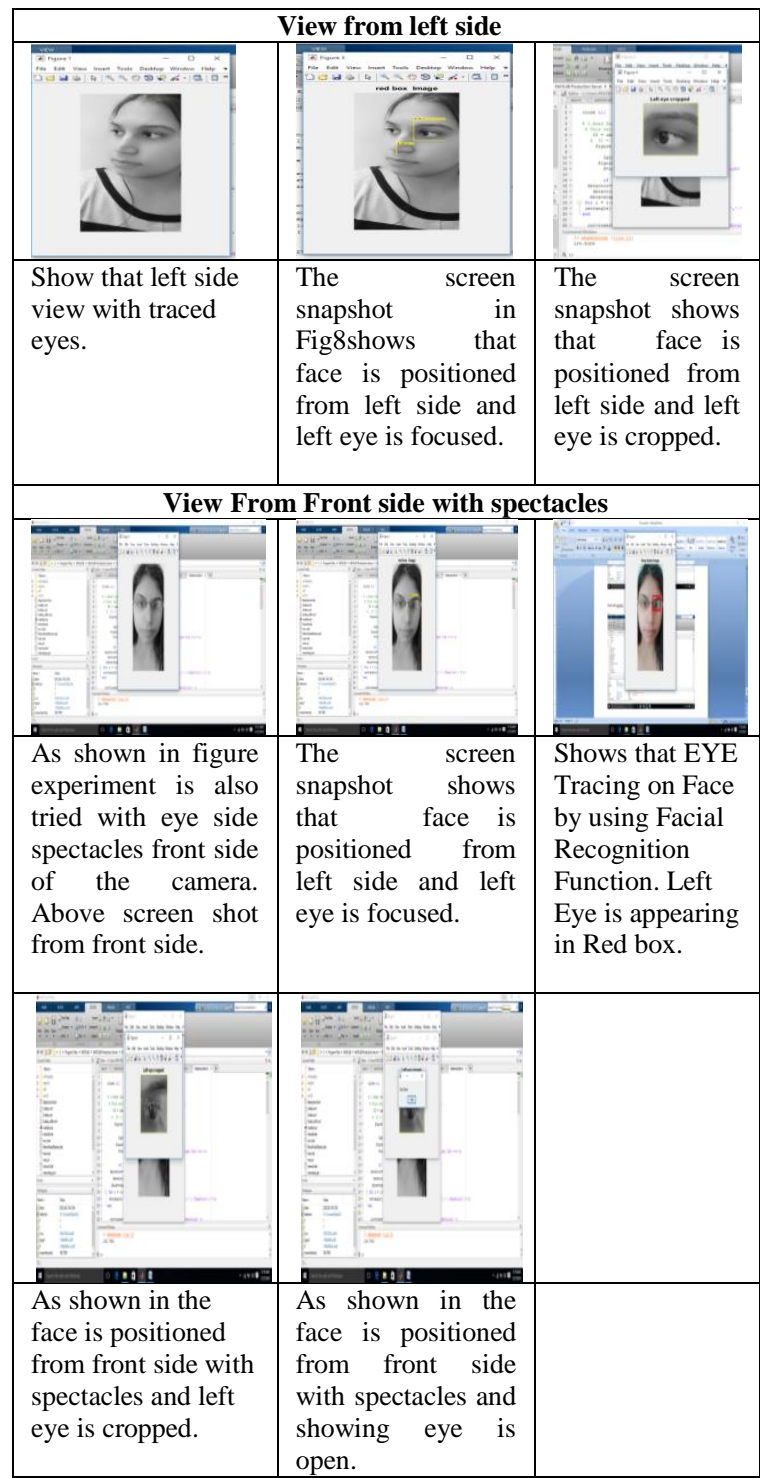

4.14 Demonstration of ProposedWork

Five sample views from different angles shown in below

\begin{tabular}{|l|l|l|}
\hline \multicolumn{3}{|c|}{ Front View } \\
\hline $\begin{array}{l}\text { Show grey scale } \\
\text { image capture } \\
\text { through android } \\
\text { mobile phone. }\end{array}$ & $\begin{array}{l}\text { Shows that EYE } \\
\text { Tracing on Face by } \\
\text { Recognition } \\
\text { Function. Both } \\
\text { Eyes are appearing } \\
\text { in Yellow boxes. }\end{array}$ & $\begin{array}{l}\text { Shows a } \\
\text { message box } \\
\text { which tells us } \\
\text { eye is open or } \\
\text { close after } \\
\text { checking } \\
\text { intensity. }\end{array}$ \\
\hline
\end{tabular}
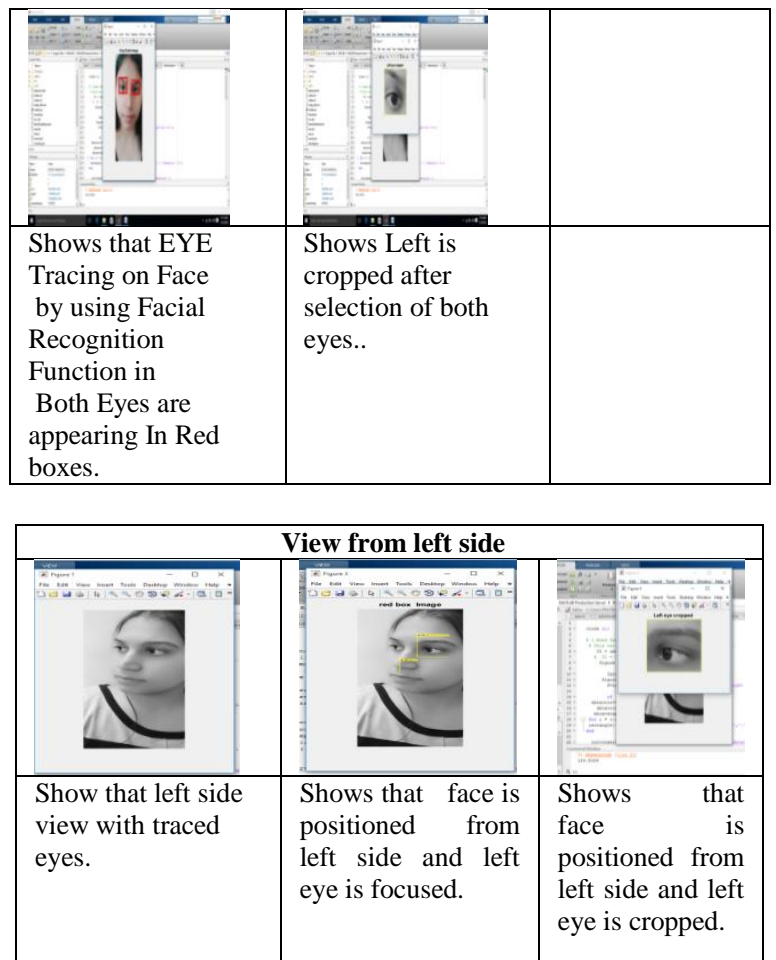

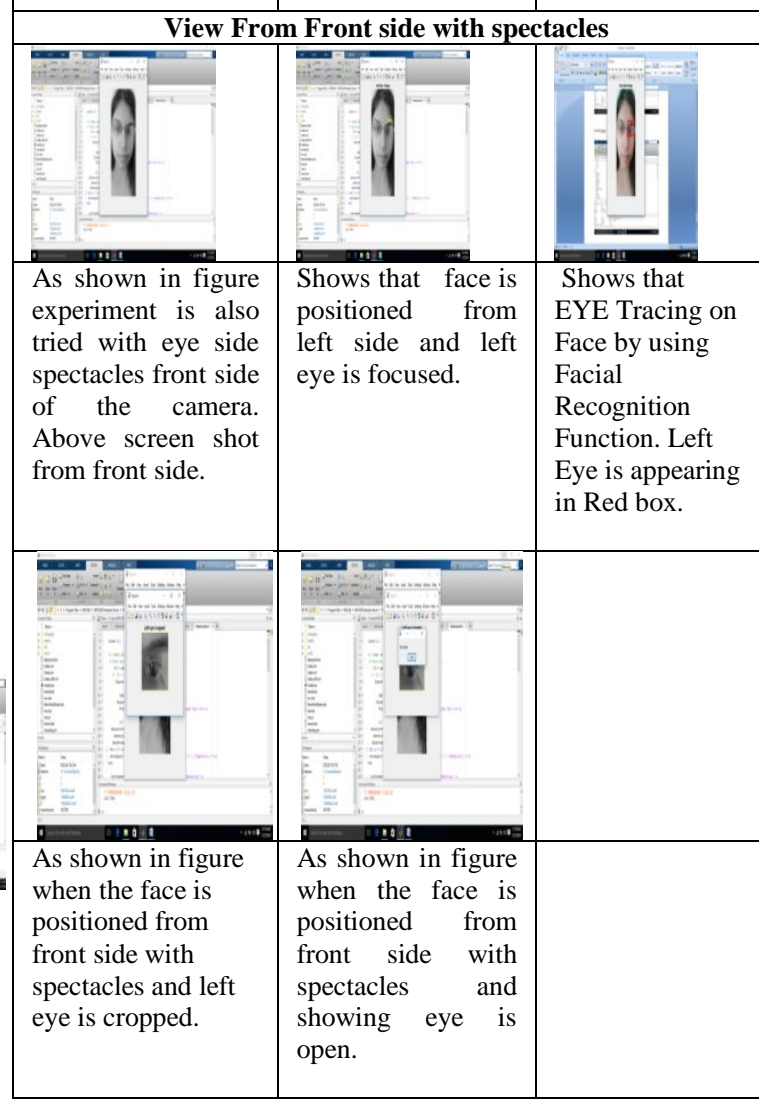

Hence proposed system is based on non-intrusive method based on visual behaviour by using digital image processing technique. Here, visual behaviour feature of eye is captured by camera. The results are taken on own created data is good and satisfactory. 
It is also tested that proposed system perform accurately with synthetic data set which were used by other systems. It is found that accuracy of proposed system was better than other systems.

\section{CONCLUSION}

The driver drowsiness system has been proposed on visual behaviour. Here, visual behaviour feature like eye block mean value computed from image, captured by Mobile Phone. The facial features and eye is detected by Paul Viola and Michael Jones algorithm. Such eye feature cropped and mean value of this eye block is computed. The dark portion of this block having low intensity value and white part of the block having high intensity value. The mean intensity value decide that eye is open or close. The accuracy of proposed system is good and satisfactory. When proposed system is tested on synthetic data (which is used by other systems) and made comparison with other methods, it is found that proposed system performance is good and satisfactory.

\section{REFERENCES}

[1] Kumari Kusuma B.M., Kumar Ramakanth P., "A survey on Drowsy Detection System", International Conference on Big Data Analytics and Computational Intelligence ,IEEE (2017), 978-1-5090-6399-4/17, page no. 272-279.

[2] Oraan Khunpisuth, et-al, "Driver Drowsiness Detection using Eye-Closeness Detection", International Conference on Signal-Image Technology \& InternetBased System", IEEE(2016), 978-1-5090-5698-9/16, page no. 661-668.

[3] Chellappa Yogesh, et-al, "Driver Fatigue Detection System”, International Conference on Signal and Image Processing,IEEE(2016), 978-1-5090-2377-6/16, page no. 655-660.

[4] Ahmed Javed, et-al, "Eye Behaviour Based Drowsiness Detection System", IEEE (2015), 978-1-4673-82663/15, page no. 268-272.

[5] Kumar Madhan A, et-al, "Drowsy Driving Warning and traffic collision information system using IoT", International Journal of Advance Research, Ideas and Innovation in Technology (2019), Volume-5, Issue-2, page no. 176-179.

[6] Viola Paul, Jones Michael, "Rapid Object Detection Using A Boosted Cascade Of Simple Features", Conference On Computer Vision And Pattern Recognition, IEEE (2001), page No. 511-518. 